\title{
Importance of patient adherence and compliance in the present day
}

Keywords: global disease, morbidity, mortality, MEMS, electronic health record

\section{Introduction}

Improving the overall global disease burden is no easy task. More so, with the number of increasing incidences of fatalities, it has become all the more important to stress upon the root causes of such conditions and treat them accordingly.

When one seeks medical attention, the first and foremost task is handed upon the physician and/or the examiner to assess the patient's medical history and then prescribe an appropriate personalized medical regimen. Be it infectious diseases, chronic conditions or metabolic disorders, prescription of medication is only the first step of proceedings. It is equally upon the patient, as much as it is upon the doctor and healthcare professionals examining him, to stick to his/her prescribed routine and get a follow-up check done on a regular basis.

In scientific terms, this refers to adherence and compliance on the patient's side. Non-compliance and non-adherence have become a rising cause of concern in modern times, because of which there has been an increase in the number of cases of resistance, morbidity and mortality.

Adherence: Adherence is defined as the degree to which a patient voluntarily integrates and collaborates with the healthcare provider in terms of instructions regarding dosage, timing and frequency of medication and gets a refill of prescriptions whenever necessary eventually leading to a better therapeutic outcome. ${ }^{1-3}$

A related term, 'medication persistence' is defined as the time from the initiation of therapy, till the time it is aborted, which might be the exact prescribed time span or a part of there..$^{1,2,4}$

Compliance: Compliance is defined as the degree to which the patient's actual regime matches that prescribed to him by the healthcare provider. ${ }^{1,5,6}$

I. Adherence and compliance are pivotal in ensuring an improved health outcome for the patient especially if he is suffering from a chronic condition and needs prolonged medical attention. Examples in this category include those with cardiovascular complications, diabetes and different forms of cancer. ${ }^{2-4}$

II. Studies have found that patients with chronic conditions, post discharge from hospitals, mostly stop adhering to their medical routines by the end of the month, with very few patients reporting to continue with medications beyond 6 to 12 months, depending on the particular class of drugs used. , $^{3,7}$

III. Increasing non-adherence has been associated with adverse health outcomes, increased rates of morbidity and mortality and increased healthcare costs.

IV. Some of the major obstacles in patient adherence include overlapping medication regimes from constantly changing
Volume 4 Issue 5 - 2017

Sreetama Dutt
The University of Manchester, United Kingdom

Correspondence: Sreetama Dutt, Scientific content writer, WEbRx Lifesystems India Pvt Ltd., Bangalore, India, Tel +919874700697, Email sreetama.dutt@gmail.com

Received: February 28, 2017| Published: May 08, 2017

healthcare providers, lack of proper education and discontinuation of medication, knowingly or unknowingly, socio-economic barriers within populations and lack of family support, social stigma and discrimination.

V. The global burden of healthcare costs has been on the rise and is projected to reach around USD 4.8trillion by 2021 in the US alone (some predicting it to be around $20 \%$ of the country's GDP in 2020), with chronic diseases accounting for threefourths of the total number of deaths globally. ${ }^{7,8}$

VI. An effective way to plummet the soaring healthcare costs is to invest a fraction of the projected healthcare costs into patient adherence and compliance programs and interventions instead of using them to come up with new medications and drug combinations, especially for patients with diabetic and cardiovascular complications, hypertension and cancer., ${ }^{2,910}$

\section{Factors related to non-adherence and non- compliance ${ }^{2,9,11,12}$}

\section{Patient-related factors}

1. Inadequate health literacy and knowledge (mostly in case of asymptomatic diseases).

2. Inadequate knowledge about the medical decision-making process.

3. Socio-economic status poses a threat towards bearing treatment costs which are often expensive and complicated.

4. Lack of communication and transportation, mostly in rural areas.

5. Lack of family support.

\section{Physician-related factors}

i. Prescribing complex medication regimes which are beyond the patient's understanding.

ii. Lack of communication between different healthcare officials (eg. Hospital officials, nurses, general physicians and consultants during post-discharge visits).

iii. Inadequate knowledge about a patient's disease background. 
iv. Inadequate knowledge about a patient's financial burden before prescribing any medication.

\section{Health system-related factors}

A. Fragmented healthcare systems, lack of co-ordination between healthcare providers, hinder a patient's access to effective health care.

B. Health information technology, though looks promising, is laced with its own loopholes which hamper effective healthcare monitoring by doctors and healthcare professionals. Healthcare IT systems need to look at the bigger picture instead of focusing on proprietary and local needs.

C. Creating an effective electronic health record (EHR) would help manage most of the shortcomings of the healthcare IT system.

D. Insufficient interaction time between healthcare providers and patients.

\section{Case studies on adherence and compliance}

Considering a study by Marin based on patients suffering from chronic myeloid leukemia (CML), the degree of impact of adherence to Imatinib reflected upon the patients' health, when analyzed on the molecular level with the help of Microelectronic Monitoring Systems (MEMS). The rates of complete cytogenic response (CCyR), major and complete molecular response (MMR and CMR respectively) showed significant improvements when patients adhered to their prescribed regimes, with minimal adverse effects. It was seen that an adherence of $\geq 90 \%$ ensured a probability of close to $90 \%$ in achieving MMR and low transcript levels of the BCR-ABL1 gene, while those with an adherence of $<90 \%$, the probability plummeting down to less than $20 \%$ when plotted graphically. Additional factors like multidrug resistance, age, levels of the molecular transporter hOCT1 and mutations in the tyrosine kinase receptor genes were also taken into account while performing this analysis. ${ }^{13}$

It was further seen that elderly patients were more compliant to the drug regimes (who had been taking Imatinib for 2years prior to the study) as compared to their younger counterparts, evident with a fivefold increase in the BCR-ABL1 transcript levels in the latter.

a. Increase in dosage however reduced adherence rates among patients.

b. The graphical representation depicting the probability of attaining MMR and CMR in adherent and non-adherent patients with respect to time is shown in the following figures. ${ }^{13}$

c. Hypertension is one of the major risk factors for developing cardiovascular diseases. In an Asian study, a group of hypertensive patients, a mix of Indians, Malayans and Chinese were assessed on the basis of a Medication Adherence Scale based on two different questionnaires - namely the Hill-Bone Adherence to Blood Pressure Therapy Scale and the 8-item Morisky Medication Adherence Scale (MMAS). Scores assigned to each patient depicted if one was adherent or not. Results revealed that patients adhering to anti-hypertensive medication had better blood-pressure control, as compared to their non-adherent counterparts. ${ }^{4,14}$

d. Since hypertension is mostly asymptomatic in initial stages, self-reporting questionnaires are beneficial in determining adherence. However, overestimation is often a complication. This study showed the average adherence rate to be $53.4 \%$, which was slightly higher than that reported by the WHO for developing countries. Also, females were inferred to be more compliant than males in this study. ${ }^{14}$

\section{Measures to increase adherence and compliance}

a. Understand the root cause of non-adherence and plan appropriate interventions and interviews to bridge the gaps. ${ }^{1,9,15}$

b. Increase patient literacy and educate patients to understand the underlying importance of adherence and compliance.

c. Self-reporting by patients, though economical and effective, have poor sensitivity and specificity. They often lead to overestimation of their adherence and compliance to the prescribed routine. It might also lead to 'stockpiling' of medication, without actually using them effectively. Hence there lies a need to supplement these patient reports in order to ensure effective utilization of the prescribed drug regimen. Studies indicate that measures involving Electronic Medication Packaging (EMP) devices (like Medication Event Monitoring Systems, MEMS) and pill counts have been proved to be more accurate and effective. ${ }^{2,11}$

d. Countries with a centralized medical database should synchronize between care givers, prescribers, dispensers and patients regarding the refill of prescriptions and then feed data into their systems, in order to have a uniform and unbiased electronic health record (HER) to ensure a patient's medication regime and compliance. A consistent review of these prescription refill records increases specificity of the process. ${ }^{1,5,16}$

e. Telemedicine is another evolving tool to bridge gaps in healthcare, and can be looked upon as a strong contender to spread awareness regarding the importance of adherence and compliance in both developing and developed countries. ${ }^{16}$

f. Educate, empower and encourage the family members and supporters to help the patient stick to his/her prescribed routine.

g. Patients under single-dose therapy, intermittent administration or are hospitalized, can have their metabolite and drug concentration in body-fluids monitored, in order to correlate their dosing frequencies. ${ }^{2,10,11,15}$

h. Patient-centric healthcare paradigms can be prescribed after assessing the patient's behavioral psychology and medical history. Be it medication or lifestyle modifications, discussing with the patient on a one-on-one basis, and mutually agreeing on a regime (the evolving concept of 'Shared Decision Making') might also be beneficial in improving patient adherence and compliance. . $^{3,410,12}$

i. Lastly, there are a set of questionnaires which help in evaluating the rates of adherence in patients, like the Eight Item Morisky Medication Adherence Scale, Hill-Bone Compliance scale, Brief Medication Questionnaire, Medication Adherence Report Scale. Combining patient interviews along with such questionnaires helps minimizing discrepancies in measurement of adherence and compliance. ${ }^{11,12}$ It is only when both sides (physician and patients) adhere and comply to the norms; one can hope to reduce the burden of diseases and lead a healthy and wholesome life. 


\section{Acknowledgements}

None.

\section{Conflict of interest}

The author declares no conflict of interest.

\section{References}

1. American Pharmacists Association. Adherence vs. persistence. 2015.

2. Ho PM, Bryson CL, Rumsfeld JS. Medication adherence. Circulation. 2009;119(23):3028-3035.

3. Agras WS. Understanding compliance with the medical regimen: The scope of the problem and a theoretical perspective. Arthritis Rheum. 1989;2(3):A2-A7.

4. Kamran A, Sadeghieh Ahari S, Biria M, et al. Determinants of patient's adherence to hypertension medications: application of health belief model among rural patients. Ann Med Health Sci Res. 2014;4(6):922927.

5. http://www.nets.nihr.ac.uk/_data/assets/pdf_file/0007/81394/ES-081412-076.pdf

6. Fraser S. Concordance, compliance, preference or adherence. Patient Prefer Adherence. 2010;4:95-96.
7. https://www.cognizant.com/whitepapers/medication-adherence-in-thereal-world-codex 1089.pdf

8. https://www.cognizant.com/InsightsWhitepapers/Predicting-PatientAdherence-Why-and-How.pdf

9. http://www.who.int/chp/knowledge/publications/adherence_full_report. pdf

10. Atreja A, Bellam N, Levy SR. Strategies to enhance patient adherence: making it simple. Medscape Gen Med. 2005;7(1):4.

11. Lam WY, Fresco P. Medication adherence measures: An overview. BioMed Res Int. 2015;2015:e217047.

12. Elwyn G, Frosch D, Thomson R, et al. Shared decision making: a model for clinical practice. J Gen Intern Med. 2012;27(10):1361-1367.

13. http://ascopubs.org/doi/pdf/10.1200/JCO.2009.26.3087

14. Ramli A, Ahmad NS, Paraidathathu T. Medication adherence among hypertensive patients of primary health clinics in Malaysia. Patient Prefer Adherence. 2012;6:613-622.

15. http://www.eu-patient.eu/globalassets/policy/adherence-complianceconcordance/adherence-paper-final-rev_external.pdf

16. http://apps.who.int/iris/bitstream/10665/44497/1/9789241564144_eng. pdf 